\title{
RUNGE FAMILIES AND INCREASING UNIONS OF STEIN SPACES
}

\author{
BY ANDREW MARKOE
}

\author{
Communicated by I. M. Singer, April 19, 1976
}

The general question of whether an increasing union of Stein spaces is itself Stein has been open for some time. Recently J. Fornaess has produced a counterexample [1]. Here a complete answer is given.

It has been known that if each pair in the union form a Runge pair, then the question has an affirmative answer. However the union may be Stein without each pair being Runge, since it is also known that the conjecture is valid for Riemann domains spread over Stein manifolds.

On the other hand, the following generalization of the Runge concept to the entire family does characterize those families which answer the question affirmatively.

Definition. An increasing family of analytic spaces $\cdots \subset X_{n} \subset X_{n+1}$ C $\cdots$ in which each $X_{n}$ is open in $X_{n+1}$ is said to be a Runge family if for every compact $K \subset X_{n}$, there exists $j$ such that $K \subset X_{j}$ and $\mathcal{O}\left(\bigcup X_{n}\right)$ uniformly approximates $O\left(X_{j}\right)$ on $K$.

Then we have

THEOREM. If $\cdots \subset X_{n} \subset X_{n+1} \subset \cdots$ is an increasing family of Stein spaces, each $X_{n}$ open in $X_{n+1}$, then the following are logically equivalent:

1. $\cup X_{n}$ is Stein.

2. $\cdots \subset X_{n} \subset X_{n+1} \subset \cdots$ is a Runge family.

3. $H^{1}\left(\bigcup X_{n}, O\right)=0$.

The proof will appear elsewhere, but the idea is to first note that $1 \Rightarrow 3$ trivially. Then the implication $3 \Rightarrow 2$ is proved using duality on complex spaces together with a Hahn-Banach style of argument. Finally $2 \Rightarrow 1$ is just a modification of the usual cohomology approximation theorem used to prove that the union is Stein when each pair is Runge.

Above it was mentioned that when the union is a Riemann domain over a Stein manifold, then the answer to the question is affirmative. This is usually proved via pseudoconvexity techniques. However, H. Laufer [2] has proved (essentially) that any increasing family of open sets in a Stein manifold is a Runge

AMS (MOS) subject classifications (1970). Primary 32E10, $32 \mathrm{E} 15$.

Key words and phrases. Stein spaces, Runge families, Riemann domains, duality on complex spaces. 
family. Thus the result for schlicht Riemann domains appears as a corollary of the theorem.

\section{REFERENCES}

1. J. E. Fornaess, An increasing sequence of Stein manifolds whose limit is not Stein, Math. Ann. (submitted).

2 H. B. Laufer, On Serre duality and envelopes of holomorphy, Trans. Amer. Math. Soc. 128 (1967), 414-436. MR 36 \#5393.

DEPARTMENT OF MATHEMATICS, UNIVERSITY OF CONNECTICUT, STORRS, CONNECTICUT 06268 